\title{
The exterior of the universe and the interior of a black hole
}

\author{
Open Physics Collaboration*† \\ March 4, 2020
}

\begin{abstract}
We propose that "outside the universe = inside a black hole".
\end{abstract}

keywords: universe, big bang, black hole, topology, logic, boundary

\section{Introduction}

1. Two cosmic events might be inherently correlated, namely, Black Holes $(\mathrm{BH})$ and the Big Bang (BB).

2. The Big Bang is the very creation of space and time.

3. Black holes are (probably) the very end of space and time [1] 3$]$.

4. Both $\mathrm{BH}$ and $\mathrm{BB}$ have a boundary.

5. The boundary of a black hole is its event horizon.

6. Assume the universe is spherical with a certain radius.

7. The boundary of the big bang is the surface at its radius.

*All authors with their affiliations appear at the end of this paper.

${ }^{\dagger}$ Corresponding author: mplobo@uft.edu.br| Join the Open Physics Collaboration 


\section{Axioms, Premises and Theorems}

8. Let $A, B, C, \ldots$ be mathematical formulas that prove a physical theory, theoretically or experimentally.

9. The formulas in (8) are either axioms or theorems within the physical theory.

10. In the following, we state the meaning of (8).

\section{Axioms}

11. $B$ : the big bang event (axiom 1 )

12. $Z$ : radius of the universe (axiom 2 )

13. $S(x)$ : spacetime at $x \in \mathbb{R}($ axiom 3$)$

14. $H(r)$ : the event horizon of a black hole with radius $r$ (axiom 4 )

\section{Premises and Definitions}

15. $X \equiv(\exists x \neg S(x))$ : there exists at least one mathematical object/structure with no spacetime dimensions (premise 5 )

16. $Y \equiv\left(\exists x_{1}\left(\left(x_{1}>Z\right) \wedge \nexists S\left(x_{1}\right)\right)\right)$ : there is no spacetime outside the universe (premise 6$)$

17. $L \equiv\left(\forall x_{2}<r\left(\nexists S\left(x_{2}\right)\right)\right)$ : there is no spacetime inside the black hole $[1-3]$ (premise 7)

18. L stands for hollow black hole [1]

19. $N(x)$ : nature of the mathematical object/structure with no spacetime dimensions (definition 8) 


\section{Theorem}

20.

$$
\begin{aligned}
& \vdash B \\
& \vdash Z \\
& \vdash S(x) \\
& \vdash H(r) \\
& \vdash X \\
& \vdash Y \\
& \vdash L \\
& \hline \vdash \nexists x((x<r) \wedge(x>z)) \\
& \vdash N\left(x_{1}\right) \equiv N\left(x_{2}\right)
\end{aligned}
$$

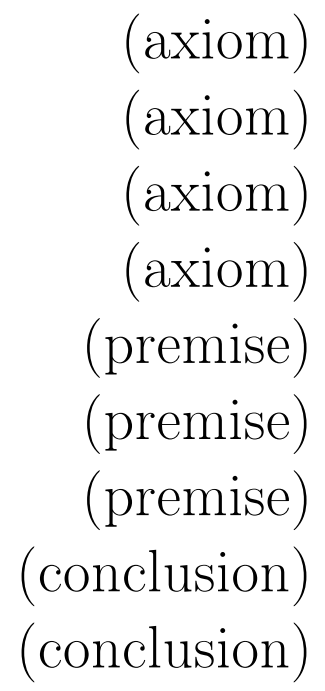

21. Let $P \equiv(B \wedge Z \wedge S(x) \wedge H(r) \wedge X \wedge Y \wedge L)$.

22. Let $Q_{1} \equiv(\nexists x((x<r) \wedge(x>z)))$.

23. Let $Q_{2} \equiv\left(N\left(x_{1}\right) \equiv N\left(x_{2}\right)\right)$.

24 .

$$
P \vdash Q_{1} \wedge Q_{2} \quad \square
$$

\section{Final Remarks}

25. What is inside a black hole and outside the universe? Are there mathematical objects/structures?

26. If the Big Bang theory is correct, then there might exist some mathematical structure with neither spatial nor time dimensions.

27. The border of the universe, if it exists, is a boundary that at one side, spatial and time dimensions can coexist, while at the other "side", there is no space and time dimensions at all. 
28. In simple words, we conclude that, what is "outside" the universe is the same as what is "inside" a black hole.

\section{Open Invitation}

Review, add content, and co-author this paper [4,5]. Join the Open Physics Collaboration. Send your contribution to mplobo@uft.edu.br.

\section{Ethical conduct of research}

This original work was pre-registered under the OSF Preprints [6], please cite it accordingly |7|. This will ensure that researches are conducted with integrity and intellectual honesty at all times and by all means.

\section{References}

[1] Lobo, Matheus P. "Hollow Black Holes." OSF Preprints, 12 Aug. 2019. https://doi.org/10.31219/osf.io/rewub

[2] Lobo, Matheus P. "The Interior of a Black Hole and the Void of Spacetime." OSF Preprints, 12 May 2019. https://doi.org/10.31219/osf.io/awfx8

[3] Lobo, Matheus P. "Black Hole Universe: A Grand Cosmic Recycler and Big Bang Generator?." OSF Preprints, 12 Aug. 2019. https://doi.org/10.31219/osf.io/pbdn3

[4] Lobo, Matheus P. "Microarticles." OSF Preprints, 28 Oct. 2019. https://doi.org/10.31219/osf.io/ejrct

[5] Lobo, Matheus P. "Simple Guidelines for Authors: Open Journal of Mathematics and Physics." OSF Preprints, 15 Nov. 2019. https://doi.org/10.31219/osf.io/fk836 
[6] COS. Open Science Framework. https://osf.io

[7] Lobo, Matheus P. "The Exterior of the Universe and the Interior of a Black Hole." OSF Preprints, 28 Nov. 2019. https://doi.org/10.31219/osf.io/huam4

\section{The Open Physics Collaboration}

Matheus Pereira Lobo (lead author, mplobo@uft.edu.br), 1,2

Marcos Vinícius Matos de Sousa, ${ }^{1}$ Ronaldo Silva Rêgo ${ }^{3}$

${ }^{1}$ Federal University of Tocantins (Brazil); ${ }^{2}$ Universidade Aberta (UAb, Portugal); ${ }^{3}$ Universidade Estadual da Região Tocantina do Maranhão 